\title{
User-Based Collaborative Filtering Using Agglomerative Clustering on Recommender System
}

\author{
Malim Muhammad \\ \{malim.muhammad@gmail.com\}
}

Department of Mathematics Education, Faculty of Teacher Training and

Education, Universitas Muhammadiyah Purwokerto

\begin{abstract}
Content-based, collaborative filtering, demographic, knowledge-based, and hybrid recommender systems are the five categories of recommendation systems. Userbased collaborative filtering and item-based collaborative filtering are the two types of collaborative filtering. However, the user-based approaches can be claimed to represent the user; researchers will employ them here. This method is more concerned with the user's likeness, or similarity than with the user's evaluated item. The accuracy of user-based collaborative filtering approaches employing agglomerative Clustering with similarity computations, i.e., cosine similarity, is improved in this study. MovieLens (https://grouplens.org/datasets/movielens/) provided the researchers with the data they needed. Between January 9, 1995, and October 16, 2016, a total of 100004 ratings for 9,125 films were collected from 671 individuals. At least 20 movies have been rated by each user. Each rating has a value of 1 to 5 . The data utilized for testing is five value data from each user. In other words, 3,355 data points were tested in total. Using the single linkage clustering approach to cluster films in the use-based method has been shown to improve the accuracy of results that differ significantly between scenarios one and two, namely 3,409 and 3.26. MAE and RMSE are the accuracy gauges utilized in the analysis, and the smaller the value (closer to zero), the better the program results. The findings of two trials (2 Scenarios) revealed significant differences between scenario 1 and scenario 2, namely 3,409 and 3.26. This is because in scenarios 1 and 2, only neighbors with similarity values greater than zero are utilized to find predictions, regardless of whether the neighbor has scored the film to be forecasted or not. In scenario 1, however, the results produced by adding the single linkage clustering approach to the user-based method as mentioned above are not as good. As the value obtained grows larger, the system's level of accuracy decreases. However, the results achieved in scenario 2 are smaller, but the differences are not significant.
\end{abstract}

Keywords: Recommended System, User-Based Collaborative Filtering, Agglomerative Clustering

\section{Introduction}

Advances in technology have made the digital search easier. Over time various sites that use search engines, be it selling sites or other sites, also use a recommendation system. The recommendation system can be used in various areas, such as movies, news, music, books, and others. According to Casey (2014), the recommendation system utilizes the history of user behavior such as articles that have been read, products that have been assessed or purchased, 
music that is often played, and so on to identify the user's preferences which are then used as references to produce final recommendations in the form of items or products [1]. The recommendation system works based on previously stored user information. This information itself can be a numeric value, an ordinal value, or a binary value [2].

According to Ricci et al. (2015), the recommendation system is divided into five types: content-based, collaborative filtering, demographic, knowledge-based, and hybrid recommender system [2]. The general principle of content-based filtering is to identify common traits of an item that gets a high rating from the user and recommend that using the item's characteristics. Collaborative filtering uses assessment information from users and other goods. Demographics recommend items based on the demographic profile or region of that user. Knowledge-based systems recommend goods based on specific information of how much an item meets needs and is useful to users. In comparison, the Hybrid Recommender System is a combination of the recommendation systems above.

Collaborative filtering is divided into two types: user-based collaborative filtering and itembased collaborative filtering [3]. However, researchers will use user-based methods because this method can be said to represent the user. This method pays more attention to the similarity or similarity of the user than the item that the user has assessed. In contrast, item-based pay more attention to the assessment of goods. This method is included in the neighborhood model that directly uses stored assessments to predict.

Some of the advantages of using neighborhood-based methods [2] are

1) Simplicity, neighborhood-based methods are relatively easy to implement

2) Justifiability, this method also provides a concise and intuitive basis of truth on the computing of its predictions.

3) Efficiency, one of the advantages of this method is its efficiency. Because this method does not require pre-computing and storage for data, its determination is not too large.

4) Stability, this method is not unduly affected by additional users, items, and ratings.

The several recommendations contained in this method, there are also shortcomings in the scope of its recommendations [2]. This becomes more visible when the data used has a fairly high sparsity. This will then reduce the scalability of this approach itself. There has been a lot of research done to improve the accuracy performance of collaborative filtering. Leben (2008) uses adjusted cosine to calculate similarity. The comparison of both collaborative filtering methods has been made by Sarwar et al. (2001) using adjusted cosine to calculate similarity on item-based and Pearson correlation on user-based [4]. The result obtained is a user-based method with Pearson correlation has higher accuracy. Based on the above, the author will try to contribute to improving the accuracy of user-based collaborative filtering methods using agglomerative Clustering with similarity calculations, i.e., cosine similarity.

A recommendation system is a technique that advises or suggests goods that are in demand by certain users [2]. There are three main processes of this technique, namely: object data collections and representations, similarity decisions, and recommendation computation. Collaborative filtering methods collect and analyze large amounts of information about a user's behavior, activity, or preferences and predict what users will like. This method does not rely on content that can be analyzed. Therefore, this method can recommend complicated items such as movies without understanding the movie itself. This is the advantage of collaborative filtering methods. One of the best-known examples of collaborative filtering is item-to-item or itembased (people who buy $\mathrm{x}$ and buy $\mathrm{y}$ ), an algorithm popularized by the recommendation system Amazon.com [5]. Last.fm recommends music based on comparisons of the same user's listening habits, while Readgeek compared book ratings to recommendations. Facebook, MySpace, LinkedIn, and other social networks use collaborative filtering to recommend friends, groups, 
and other social connections (by checking the network of connections between users and their friends). Twitter uses a lot of signals and calculations in memory to recommend to its users who to follow [2],[6].

In collaborative filtering, there is also a user-based method, where this method will recommend goods to users $\mathrm{x}$, which is also liked by other users similar to $\mathrm{x}$ [2]. So, between the user-based method and the item-to-item method, it is almost the same. However, the difference lies in what the recommendations are. User-based sees the user's resemblance to other users, while item-based looks at it in terms of goods.

Agglomerative Clustering has a way of working with the assumption that $\mathrm{n}$ items want to be clustered and matrix distance or similarity $\mathrm{N} * \mathrm{~N}$. The basic process, according to Johnson (1967), is as follows:

1) Create clusters for each item so that if it has $n$ items, it will now have $n$ clusters, which contain one item. Let the similarity between clusters equal the similarity between the items in it.

2) Find the nearest (most similar) cluster pair and combine the two into one cluster, so we now have one fewer cluster

3) Calculate the similarity between the new cluster and each of the old clusters.

4) Repeat steps 2 and 3 until all items are grouped into one group with size $\mathrm{N}$

Step 3 can be done in several different ways, namely minimum proximity, maximum proximity, average proximity, and centroid proximity. At minimum proximity (single-linkage), the shortest distance (largest similarity) is used to create clusters, while at maximum proximity (double-linkage) used is the largest distance (smallest similarity).

Pearson Correlation Coefficient and Cosine Similarity are commonly used and well-known similarity methods. That's why I use both methods. Pearson correlation coefficient will be used for calculation of final prediction of goods (recommendations) and cosine similarity for similarity calculations to create clusters.

The similarity is a method in machine learning that serves to calculate similarities between 2 or more data. Similarity calculations use algorithmic methods such as Pearson correlation, cosine similarity, and many more. Pearson Correlation is one of the algorithms commonly used in calculating the similarity between users and other users. Correlation is a measurement technique that determines the closeness of relationships between two sets of different numbers. Correlation calculations have a condition where the set of numbers calculated must have a fixed order and pair with each other between the two sets. The results of measurement can be either positive relationships or negative relationships. Positive relationships indicate that both sets have a tendency to increase or increase equal values. In contrast, the negative relationship shows that both sets have a tendency to decrease or decrease in equal value [8]. Here is the Pearson Correlation equation for calculating the similarity between users:

$$
P C(u, v)=\frac{\sum_{i=1}^{n}\left(r_{u i}-\bar{r}_{u}\right)\left(r_{v i}-\bar{r}_{v}\right)}{\sqrt{\sum_{i=1}^{n}\left(r_{u i}-\bar{r}_{u}\right)^{2}\left(r_{v i}-\bar{r}_{v}\right)^{2}}}
$$

Where:

- $\quad P C(u, v)$ is the similarity value between user u and user $\mathrm{v}$

- $\quad r_{u i}$ and $r_{v i}$ is the user rating ui and vi to item-i 
- $\quad \bar{r}_{u}$ and $\bar{r}_{v}$ is the average rating $\mathrm{u}$ and $\mathrm{v}$ to item-i

- $\quad n$ is the number of items

Cosine similarity is a method used to calculate the similarity of each user with each other. Different rating scales between different users will result in different similarity values [8]. Here is the Cosine Similarity equation for calculating the similarity between users:

$$
\operatorname{Sim}\left(u_{i}, u_{k}\right)=\frac{r_{i} \cdot r_{k}}{\left|r_{i}\right|\left|r_{k}\right|}=\frac{\sum_{j=1}^{m} r_{i j} r_{i k}}{\sqrt{\sum_{j=1}^{m} r_{i j}{ }^{2} \sum_{j=1}^{m} r_{k j}{ }^{2}}}
$$

Where:

- $\operatorname{Sim}\left(u_{i}, u_{k}\right)$ is the similarity value between the ith user and the kth user

- $\quad u_{i}$ and $u_{k}$ is the ith user and the kth user

- $\quad r_{i}$ and $r_{k}$ is the ith user rating and the kth user

- $m$ is the number of items

Different similarity indicators will result in different prediction scores. Mean absolute error (MAE) and root mean square error (RMSE) are two common indicators for measuring the accuracy of similarity methods. The smaller the value, the better the prediction accuracy. It is defined as follows:

1) Mean absolute error (MAE) is the average of the absolute error of the user's predicted score and the true score in the scoring test set $q_{i} p_{i}$.

$$
M A E=\frac{\sum_{i=1}^{n}\left|p_{i}-q_{i}\right|}{n}
$$

2) Root-mean-square error (RMSE) is the mean square root of the true score value and the predicted score value of the user in the test set: $p_{i} q_{i}$

$$
R M A E=\sqrt{\frac{\sum_{i=1}^{n}\left(p_{i}-q_{i}\right)^{2}}{n}}
$$




\section{Research Methods}

The data used by the researchers was obtained from a site called MovieLens (https://grouplens.org/datasets/movielens/). The dataset consists of 100004 ratings of 9,125 films obtained from 671 users between January 9, 1995, and October 16, 2016. Each user has rated at least 20 movies. Each rating is worth from 1 to 5 . For testing, the data used is five value data from each user. In other words, the total data testing used is 3,355 data.

The test scenario is done by conducting the experiment twice. First, implement the userbased method directly by using 100 neighbors regardless of whether the neighbor has judged the film to be predicted. Second, implement the user-based method directly by using a maximum of 100 neighbors who have assessed the film to be predicated on the implementation of Clustering. In experiments one and two, all films will be clustered first into 5 clusters because the rating value used is nominal 1 to 5 . Clustering is done using the agglomerative clustering technique (single linkage). After that, it will be implemented user-based methods in the same way in the first and second scenarios. For more details, you can see it in figure 1.

Scenario 1 and 2

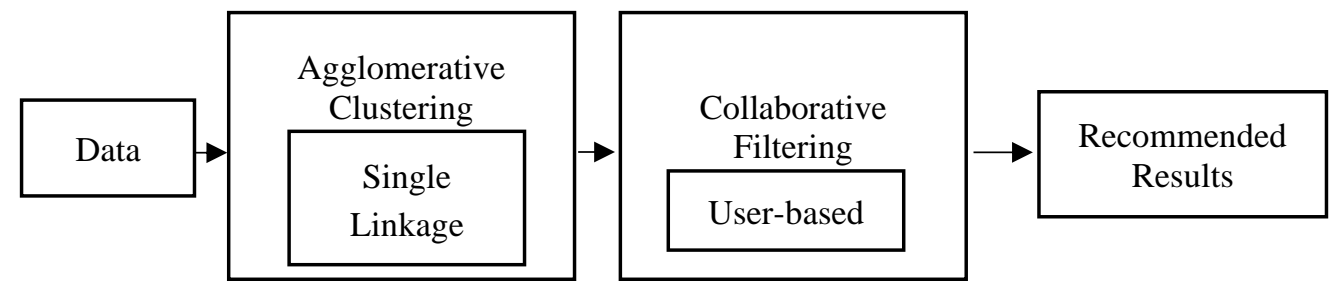

Figure 1. Scenario Illustration 


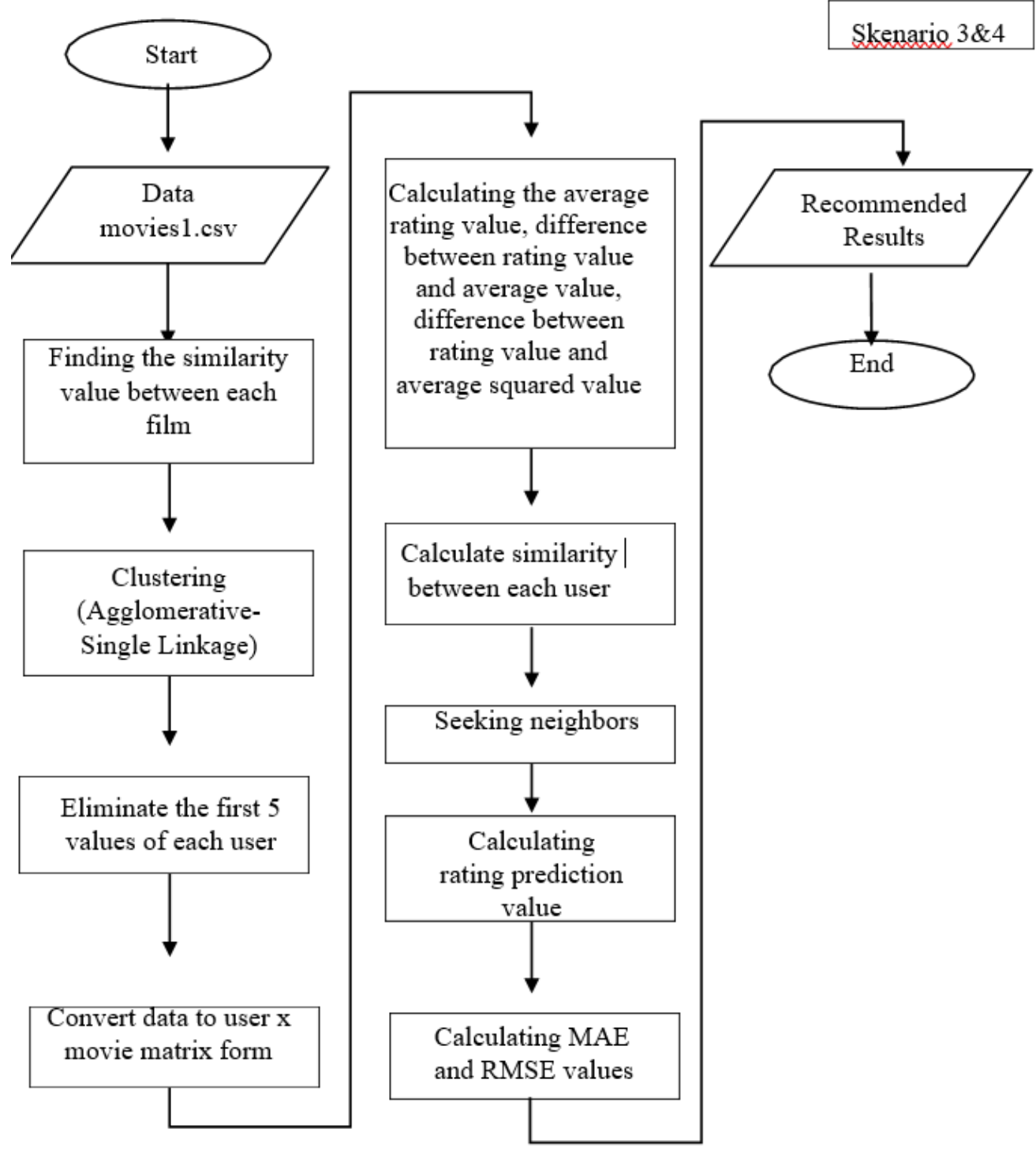

Figure 2. Flowchart process design with Clustering

\section{Results and Discussion}

The implementation of process blocks in a class is in table 1, and the implementation of process blocks in functions is in table 2. While in table 3 contains the implementation of functions in the following classes: 
Table1. Implementation of process blocks in a class

\begin{tabular}{|l|l|}
\hline \multicolumn{1}{|c|}{ Process Block } & \multicolumn{1}{c|}{ Class } \\
\hline Eliminates the first 5 values from each user & $\begin{array}{l}\text { pearson.m, pearson1.m, pearson_cluster.m } \\
\text { and pearson_cluster1.m }\end{array}$ \\
\hline Convert data to user matrix form x movie & $\begin{array}{l}\text { pearson.m, pearson1.m, pearson_cluster.m } \\
\text { and } \\
\text { pearson_cluster1.m }\end{array}$ \\
\hline $\begin{array}{l}\text { Calculate the predicted value } \\
\text { rating }\end{array}$ & $\begin{array}{l}\text { Pearson.m, pearson1.m, pearson_cluster.m } \\
\text { and } \\
\text { pearson_cluster1.m }\end{array}$ \\
\hline RMSE & $\begin{array}{l}\text { Pearson.m, pearson1.m, pearson_cluster.m } \\
\text { and } \\
\text { pearson_cluster1.m }\end{array}$ \\
\hline Find the similarity value between each movie & nilai_cosine.m \\
\hline Clustering (Agglomerative - Single Linkage) & AHC.m \\
\hline
\end{tabular}

Table2. Implementation of process blocks on functions

\begin{tabular}{|l|l|}
\hline \multicolumn{1}{|c|}{ Process Block } & \multicolumn{1}{c|}{ Function } \\
\hline Calculate the average rating & m_nilaiRata2 \\
\hline $\begin{array}{l}\text { the difference in rating value with the } \\
\text { average value }\end{array}$ & m_rai \\
\hline $\begin{array}{l}\text { the difference in rating value with the } \\
\text { average square }\end{array}$ & m_raikuadrat \\
\hline Hitung similarity & m_pearson \\
\hline Looking for neighbors & finding_topN \\
\hline
\end{tabular}

Table3. Implementation of functions in the class

\begin{tabular}{|l|l|}
\hline Function & \multicolumn{1}{|c|}{ Class } \\
\hline m_nilaiRata2 & $\begin{array}{l}\text { pearson.m, pearson1.m, pearson_cluster.m and } \\
\text { pearson_cluster1.m }\end{array}$ \\
\hline m_rai & $\begin{array}{l}\text { pearson.m, pearson1.m, pearson_cluster.m and } \\
\text { pearson_cluster1.m }\end{array}$ \\
\hline m_raikuadrat & $\begin{array}{l}\text { pearson.m, pearson1.m, pearson_cluster.m and } \\
\text { pearson_cluster1.m }\end{array}$ \\
\hline m_pearson & $\begin{array}{l}\text { pearson.m, pearson1.m, pearson_cluster.m and } \\
\text { pearson_cluster1.m }\end{array}$ \\
\hline finding_topN & $\begin{array}{l}\text { pearson.m, pearson1.m, pearson_cluster.m and } \\
\text { pearson_cluster1.m }\end{array}$ \\
\hline
\end{tabular}

The detailed usability of each class is in table 4 , while table 5 contains the usability of each function in the system. 
Table 4. Class and its uses

\begin{tabular}{|c|c|}
\hline Class & Uses \\
\hline Pearson & $\begin{array}{l}\text { Search for rating predictions using } 100 \text { neighbors without seeing } \\
\text { if the neighbor has passed the movie. } \\
\text { It will be predicted or not. }\end{array}$ \\
\hline Pearson1 & $\begin{array}{l}\text { Search for rating predictions using a maximum of } 100 \text { neighbors } \\
\text { who have rated the film to be predicted }\end{array}$ \\
\hline Pearson_cluster & $\begin{array}{l}\text { Search for rating predictions using } 100 \text { neighbors without seeing } \\
\text { if the neighbor has passed the film to be predicted or not by } \\
\text { implementing agglomerative Clustering }\end{array}$ \\
\hline Pearson1_cluster & $\begin{array}{l}\text { Search for rating predictions using a maximum of } 100 \\
\text { neighbors who have rated the film to be predicted by } \\
\text { implementing agglomerative Clustering }\end{array}$ \\
\hline Nilai_cosine & Search for the cosine similarity value of all movies \\
\hline $\mathrm{AHC}$ & $\begin{array}{l}\text { Useful for clustering movies using techniques } \\
\text { agglomerative clustering }\end{array}$ \\
\hline
\end{tabular}

Table5. Its functions and uses

\begin{tabular}{|l|l|}
\hline \multicolumn{1}{|c|}{ Function } & \multicolumn{1}{c|}{ Uses } \\
\hline AND & Useful for finding weight values between two films \\
\hline m_nilaiRata2 & Search for the average rating of each user \\
\hline m_rai & Find the difference in rating value by the average value \\
\hline m_raikuadrat & $\begin{array}{l}\text { Searches for the difference in the value of the rating with the } \\
\text { average square. }\end{array}$ \\
\hline m_pearson & Search for Pearson value(similarity)between all users \\
\hline finding_topN & $\begin{array}{l}\text { Search for the 100 neighbors with the greatest similarity value of } \\
\text { all users }\end{array}$ \\
\hline cari_cluster & Search for movie clusters to predict \\
\hline cari_user & $\begin{array}{l}\text { Search for users - users who have rated movies in the movie } \\
\text { cluster to predict }\end{array}$ \\
\hline
\end{tabular}

Class pearson_cluster (scenario 1)

In the pearson_cluster class will be used a user-based method by applying agglomerative Clustering (single linkage). Where the steps to cluster the entire film have been done first.

1) Read data from ratings1.csv.

2) Eliminate the first five ratings from each user that will be used for data testing.

3) Convert to the form of user matrix $x$ movie.

4) Look for the movie cluster to predict and the users-users who have ranked the movie-the movie that is in the cluster. Then convert the data into the form of a user matrix $\mathrm{x}$ movie.

5) Calculate the average rating of each user, the difference in the rating value with the average value, the difference in the rating value with the average value of the square.

6) Calculates the similarity between each user using the Pearson correlation algorithm

7) Search for 100 neighbors of all users using the similarity value you've searched for

8) Look for the user index and the movie to predict. This is because the presence of users and movies that will be predicted cannot match the value of rows or columns. 
9) Perform a neighbor selection process to calculate a user's rating prediction of a movie (out of 100 neighbors of that user, will be used whose similarity value is only large from zero)

10) Then calculate the rating prediction using the user-based method. In a way, all neighbor ratings are multiplied by the similarity value, then divided by the same amount of the entire neighborhood. This step will continue until all data testing has been predicted.

11)Calculate MAE and RMSE to determine the system's error value or accuracy rate after all the testing data is calculated or predicted.

Class pearson_cluster1 (scenario 2)

The steps in this class are nearly identical to those in the Pearson cluster class. It's only that this class has a different approach to selecting neighbors. The neighbors sought are the users who will be predicted's neighbors, with a maximum of 100 persons, each of whom has judged the movie to be forecasted. MAE and RMSE are the accuracy gauges utilized in the analysis, and the smaller the value (closer to zero), the better the program results. From two trials (two situations), a significant difference was found between scenario one and scenario 2, namely 3,409 and 3.26. This is because, in scenarios 1 and 2, only neighbors with similarity values greater than zero are utilized to check for predictions, regardless of whether the neighbor has scored the movie to be forecasted or not. In scenario 1 , however, the results produced by adding the single linkage clustering approach to the user-based method as mentioned above are not as good. As the value obtained grows larger, the system's level of accuracy decreases. However, the results produced in scenario two do get smaller, but the differences are not significant.

\section{Conclusion}

The employment of the single linkage clustering approach to cluster movies using the usebased method has been shown to enhance the accuracy of findings that differ significantly between scenarios 1 and 2, namely 3,409 and 3.26. MAE and RMSE are the accuracy gauges utilized in the analysis, and the smaller the value (closer to zero), the better the program results. From two trials (two situations), a significant difference was found between scenario one and scenario 2, namely 3,409 and 3.26. This is because, in scenarios 1 and 2, only neighbors with similarity values greater than zero are utilized to check for predictions, regardless of whether the neighbor has scored the movie to be forecasted or not. In scenario 1, however, the results produced by adding the single linkage clustering approach to the user-based method as mentioned above are not as good. As the value obtained grows larger, the system's level of accuracy decreases. However, the outcomes achieved in scenario 2 grow smaller, although the changes are not too significant.

Acknowledgments. The authors would like to express their gratitude to Universitas Muhammadiyah Purwokerto for allowing us to assist and support in completing this research.

\section{References}

[1] Casey, E. 2014. Scalable Collaborative Filtering Recommendation Algorithms on Apache Spark. Claremont: CLAREMONT McKENNA COLLEGE.

[2] Ricci, F., Rokach, L. \& Shapira, B. 2015. Recommender Systems Handbook (second edition). New York: Springer Science+Business Media LLC.

[3] Schafer, J.B., Frankowski, D., Herlocker, J., \& Sen, S. 2007. Collaborative Filtering Recommender Systems. Dalam Brusilovsky, P., Kobsa A. \& Nejdl, W. The Adaptive Web (hlm. 291-324). Berlin: Springer. 
[4] Sarwar, B., Karypis, G., Konstan, J., \& Riedl, J. 2001. Item-based collaborative filtering recommendation algorithms. Proceedings Konferensi internasional ke-10 dari World Wide Web, halaman 285-295, New York, NY, USA. ACM.

[5] Linden, dkk. 1998. Collaborative Recommendations Using Item-to-Item Similarity Mappings.

[6] Gupta, P. dkk. WTF: The who-to-follow system at Twitter. Proceedings konferensi internasional ke22 dari World Wide Web. May 13 - 17, 2013. Pages 505-514.

[7] Jhonson, K. 1967. Notes on regression and inheritance in the case of two parents.

[8] Theodorus, A., \& Budiyanto Setyohadi, D. (2016). User-Based Collaborative Filtering by Utilizing Pearson-Correlation To Find Nearby Neighbors In The Recommendation System. Master's Thesis in Information Technology, Atma Jaya University Yogyakarta, 1-6. http://e- journal.uajy.ac.id/8924/ 